\title{
Brighter cancer probes
}

\author{
The early detection of cancer demands translatable light-emitting or light-collecting probes with \\ unprecedented levels of sensitivity and specificity.
}

Diagnosing solid tumours using non-ionizing light in a minimally invasive manner can be achieved in essentially three ways: by shining light on suspected malignant tissue and analysing the reflected light, by means of a reporter probe that lights up only when taken up by tumours, or through the detection of tumour biomarkers captured from bodily fluids such as blood or urine. Endoscopy and laparoscopy are routinely used in hospitals to examine (and biopsy) tissue, yet detection of potentially abnormal tissue relies on the physician's experience. Fluorescent probes that target endogenous tumour biomarkers or nanoprobes that accumulate in tumours do not require knowledge of the location of a tumour or it being surgically accessible, yet the probes require stringent safety and efficacy requirements before they can reach the clinic. Instead, analysing a patient's blood or urine for cancer biomarkers involves less risk, but requires a priori knowledge of which biomarkers to look for. Irrespective of the advantages and disadvantages of all these approaches, they all benefit from research geared towards increasing their sensitivity (so as to minimize the proportion of missed tumours) and specificity (to avoid misdiagnosing healthy patients). Eight technological advances representative of the three approaches for diagnosing tumours are included in this focus issue on cancer diagnostics.

The best clinically available method for the identification of malignant pancreatic cysts is endoscopic ultrasound-guided fine-needle aspiration, yet its specificity is only $\sim 50 \%$. Lev Perelman and colleagues (article no. 0040) describe a fibre-optic probe, compatible with routine endoscopicaspiration procedures (see image), that distinguishes weakly scattered photons from cancer tissue over the more broadly diffuse photons from healthy tissue. In a doubleblind prospective study of 25 patients, the technique achieved an overall accuracy of $95 \%$ in cysts with definitive diagnosis (benign, precancerous or cancerous).

Probes at the nanoscale can be designed to target tumours and to emit light at wavelengths that can effectively penetrate tissue and be detected by sensors outside the body. This is the main advantage of nanoparticles that emit in the nearinfrared spectral range, in particular in

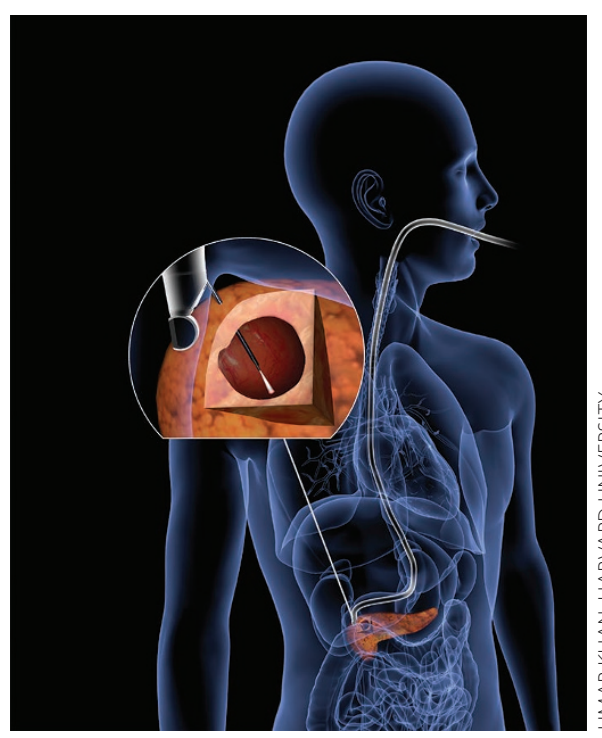

colleagues (article no. 0054), nanoparticles optimized with the right amount and type of ligands (tumour-penetrating peptides and tumour-protease-cleavable fluorescent peptides) delivered intravenously can detect tumours smaller than $2 \mathrm{~mm}$ in an orthotopic model of ovarian cancer. Because fluorescent peptides cleaved by tumour proteases filter through the kidneys into the urine, detection involves a simple urine test.

In urine, the low amounts of microRNAs and other nucleic acids secreted by cancer cells are difficult to detect by point-of-care diagnostics. Daniel Heller and collaborators (article no. 0041) show that a carbonnanotube-DNA complex with a miRNAcapture domain can sense, via blue shifts in fluorescence, miR-19 spiked into urine down to concentrations of $1 \mathrm{nM}$, and at $100-\mathrm{pmol}$ levels when the sensors were implanted in the intraperitoneal cavity of live mice. Direct detection of biomarkers in biofluids can also be achieved through signal enhancement by antibody-conjugated plasmonic nanoparticles, as Ye Hu and colleagues (article no. 0021) show with a rapid and inexpensive ultrasensitive sensor chip for capturing extracellular vesicles (EVs). By using only $1 \mu \mathrm{l}$ of blood plasma from cancer patients, the assay quantified a biomarker (identified via proteomic analyses of the content of the EVs) for pancreatic adenocarcinoma that outperformed the standard clinical biomarker. And to isolate nanoscale EVs, including exosomes, Si-Yang Zheng and co-authors (article no. 0058) took advantage of the spontaneous insertion of biotin-labelled lipid nanoprobes into vesicle membranes to isolate the vesicles in two steps and just 15 minutes via biotin-avidin binding on avidin-tagged magnetic beads. Downstream analyses of the content of the EVs allowed the authors to detect genetic mutations in plasma samples of non-small-cell lung-cancer patients.

As discussed by Catherine Alix-Panabières and Klaus Pantel in a Comment (article no. 0065), analysing exosomes alongside circulating tumour cells (CTCs) will further the understanding of metastasis. In fact, such 'liquid biopsies' provide an example of how biological understanding steered the clinical focus for interventional decisions from CTC counts to CTC-derived biomarkers in what was a decade-long process. The early detection of cancer can never come too soon. 